\title{
Inhibition of autophagy alleviates the senescent state of rat mesenchymal stem cells during long-term culture
}

\author{
YONG ZHENG $^{1 *}$, CHENG-JUN HU ${ }^{*}$, RU-HONG ZHUO ${ }^{2}$, YUE-SHAN LEI $^{1}$, NA-NA HAN ${ }^{3}$ and LIU HE ${ }^{1}$ \\ ${ }^{1}$ Department of Anatomy and Embryology, Wuhan University School of Medicine; ${ }^{2}$ Department of Integrated Medicine, \\ Zhongnan Hospital of Wuhan University; ${ }^{3}$ Department of Endodontics, School of Stomatology, \\ Wuhan University, Wuhan, Hubei 430071, P.R. China
}

Received November 24, 2013; Accepted July 2, 2014

DOI: $10.3892 / \mathrm{mmr} .2014 .2624$

\begin{abstract}
Following a limited number of cell divisions, mesenchymal stem cells (MSCs) undergo senescence, and these senescent cells maintain metabolic modification and remain viable for long periods. Autophagy, an intracellular bulk degradation process, provides a survival effect for cells under stress. In this study, the effect of autophagy on senescent MSCs was analyzed. Following serial passaging, rat MSCs underwent replicative senescence, characterized by positive staining for senescence-associated $\beta$-galactosidase (SA- $\beta$-gal), and increased expression levels of p16 and p21. During MSC senescence, the levels of autophagic activity were increased, a greater number of autophagic vacuoles were observed in senescent MSCs by transmission electron microscopy, acridine orange staining was elevated and the expression levels of autophagy-related proteins (microtubule-associated protein 1A/1B-light chain 3-II, Atg7 and Atg12) were increased. The role of autophagy in MSC senescence was further investigated through pharmacological inhibition of autophagy with bafilomycin A1 and 3-methyladenine. Inhibition of autophagy by pharmacological means reduced the rate of positive staining for SA- $\beta$-gal and the expression levels of senescence-related proteins. In conclusion, these findings suggest that autophagy is activated during senescence and the autophagic activity may be a requirement for maintaining the senescent state of MSCs.
\end{abstract}

\section{Introduction}

Mesenchymal stem cells (MSCs), the most promising autologous cell source, show great promise in various clinical

Correspondence to: Dr Cheng-Jun Hu, Department of Anatomy and Embryology, Wuhan University School of Medicine, 185 Donghu Road, Wuhan, Hubei 430071, P.R. China

E-mail: chengjunhu@whu.edu.cn

*Contributed equally

Key words: senescence, autophagy, mesenchymal stem cells, autophagy inhibitor applications $(1,2)$. However, MSCs in bone marrow are rare populations that require extensive in vitro expansion prior to clinical use. Futhermore, MSCs reach replicative senescence subsequent to a certain number of cell divisions as normal somatic cells. The senescent cells develop an enlarged and flat senescent morphology and ultimately stop division. In addition, senescent MSCs exhibit reduced differentiation potential, which limits the application of MSCs in therapeutic and tissue engineering (3).

Senescent cells are known to remain viable for long periods and secrete large quantities of proteins, which reinforces the senescence phenotype and establishes the senescence-associated secretory phenotype (4). This protein remodeling is coupled with another cell process, autophagy (5), which is an evolutionarily conserved mechanism mediating the turnover of a diverse set of cytoplasmic components, including abnormal intracellular proteins and damaged organelles (6). Previous studies have indicated that changes in the activity of the autophagic system are involved in numerous age-related diseases, such as Alzheimer's, Parkinson's and Huntington's disease (7).

Autophagy and senescence are two closely associated biological phenomena, which may be induced through external or internal forms of stress, including DNA damage, radiation, chemotherapy and telomere shortening (8). Recently, Goehe et al (9) reported that autophagy and senescence are regulated by overlapping signaling pathways involving the generation of reactive oxygen species, induction of p53 and p21, and dephosphorylation of retinoblastoma protein. However, the potential roles of autophagy during senescence remain unclear.

\section{Materials and methods}

Cell culture. Animal experimental procedures were approved by the Institutional Animal Care Committee of Wuhan University (Wuhan, China). Bone marrow MSCs were isolated from the femora and tibiae of Sprague Dawley rats (160-180 g) using a previously described method $(10,11)$. The rats were supplied by the Experimental Animal Center of Wuhan University (Wuhan, China). Briefly, following sacrifice under ether (3\%) anesthesia, the ends of the bones were cut open and the bone marrow was flushed with $\sim 4 \mathrm{ml}$ culture medium using a sterile syringe. The MSCs were seeded in Dulbecco's modified Eagle's medium supplemented with $10 \%$ fetal bovine 
serum and penicillin $(100 \mathrm{U} / \mathrm{ml}) /$ streptomycin $(100 \mathrm{mg} / \mathrm{ml})$. The culture medium was replaced once every $24 \mathrm{~h}$ three times in order to remove non-adherent cells. Subsequently, the culture medium was changed every three days. When the cells reached $80 \%$ confluence, the adherent MSCs were detached with $0.25 \%$ trypsin and passed at a ratio of 1:3.

To determine proliferative ability, the cells were seeded in three 24 -well plates $(5,000$ cells per well). After $48 \mathrm{~h}$ incubation, the cells were trypsinized to produce a single cell suspension and stained with $0.2 \%$ trypan blue. The viable cell number was counted with a hemocytometer (Qiujing Inc., Shanghai, China) under a phase-contrast microscope (CKX 41; Olympus Corporation, Tokyo, Japan). The average cell number was calculated from four wells from each of three plates and the cell counting procedure was repeated every $48 \mathrm{~h}$.

Chemical reagents. Bafilomycin A1 (Baf A1) and 3-methyladenine (3-MA), purchased from Sigma-Aldrich (St. Louis, MO, USA) and Cayman Chemical (Ann Arbor, MI, USA) respectively, were used extensively for autophagic inhibition. To produce a stock solution, $1 \mathrm{mg}$ Baf A1 and $30 \mathrm{mg} 3$-MA were dissolved in $1 \mathrm{ml}$ dimethylsulfoxide and $1 \mathrm{ml}$ double-distilled water, respectively. Future dilutions were performed in culture medium to obtain the desired concentrations. Acridine orange (A9231; Sigma-Aldrich) was used to detect autophagy.

Senescence-associated $\beta$-galactosidase assay. The cells were washed twice with phosphate-buffered saline (PBS) and stained using a senescence $\beta$-galactosidase (SA- $\beta$-gal) staining kit (Beyotime Biotech., Jiangsu, China), according to the manufacturer's instructions. Senescent cells (SA- $\beta$-gal positive cells) were counted under an inverted microscope (CKX 41SF; Olympus Corporation, Tokyo, Japan) and data were expressed as the mean percentage of total cells from five independent wells.

Acridine orange staining. To monitor autophagy morphological characteristics, the formation of acidic vesicular organelles (AVOs) was quantified by acridine orange staining (12). MSCs were washed with PBS and stained with $1 \mu \mathrm{M}$ acridine orange for $15 \mathrm{~min}$ at $37^{\circ} \mathrm{C}$. Subsequent to further washing with PBS, the cells were examined and imaged under a fluorescence microscope (BX51; Olympus Corporation). Depending on acidity, autophagic lysosomes appeared as orange/red cytoplasmic vesicles, while the nuclei were stained green.

Protein extraction and western blot analysis. Subsequent to washing in ice-cold PBS, the cultures were immediately lysed in RIPA assay lysis buffer containing protease inhibitor. Total protein concentrations were quantified with a bicinchoninic protein assay kit (P0012S; Beyotime Biotech, Jiangsu, China). For western blotting, equal quantities of protein were separated in 16\% SDS-polyacrylamide gels and electrophoretically transferred to polyvinylidene difluoride membranes. Subsequent to blocking with 5\% non-fat milk, the membranes were incubated with specific primary antibodies (1:1,000 dilutions) against microtubule-associated protein 1A/1B-light chain 3 (LC3; 4108; Cell Signaling Technology,Inc., Danvers, MA, USA), Atg7 (8558; Cell Signaling Technology, Inc.), Atg12 (4180; Cell Signaling Technology, Inc.), p16 (SC-1661, Santa Cruz Biotechnology, Inc.,
Santa Cruz, CA, USA) or p21 (BS1269; Bioworld Technology, Inc., Minneapolis, MN, USA) overnight. The membranes were then washed three times with tris-buffered saline and Tween ${ }^{\circledR}$ (TBST), and incubated with horseradish peroxidaseconjugated secondary antibody (1:3,000 dilution; BS13278, Bioworld Technology, Inc.) for $1 \mathrm{~h}$ at room temperature. Following a further three washes with TBST, the bands were visualized by enhanced chemiluminescence.

$R N A$ extraction and reverse transcription polymerase chain reaction (RT-PCR) analysis. Total RNA was isolated using TRIzol reagent (Invitrogen Life Technologies, Carlsbad, CA, USA) and reverse-transcribed to cDNA using a PrimeScript RT reagent kit (Takara Bio, Inc., Shiga, Japan). RT-PCR was performed using a SuperReal Premix [Tiangen Biotech (Beijing) Co., Ltd., Beijing, China] on the Bio-Rad IQ2 Real-Time PCR system (Bio-Rad, Hercules, CA, USA). The relative gene expression levels were calculated by the $2^{-\triangle \Delta} \mathrm{CT}$ method and the values were normalized to those of GAPDH. Each experiment was performed in triplicate. The specific primers used were: ATG7 forward: 5'-GAG GAG ACC GTC TGA GCA AC-3' and reverse: 5'-TGA CAC AGG AAA GGG TGC AA-3'; ATG12 forward: 5'-AAA CGT GAG CCA AGG GGATT-3' and reverse: 5'-GGA AAC TTG GTG CTG CTT GG-3'; GAPDH forward: 5'-GAT GGG TGT GAA CCA CGA GAAA-3' and reverse: 5'-ACG GAT ACA TTG GGG GTA GGA-3'.

Transmission electron microscopy (TEM). The cells were harvested with trypsin and fixed with $2.5 \%$ glutaraldehyde in $0.1 \mathrm{ml}$ acodylate buffer $(\mathrm{pH} 7.4)$ for $2 \mathrm{~h}$ at $4^{\circ} \mathrm{C}$. Following two washes in cacodylate buffer, the samples were post-fixed with $1 \%$ osmium tetroxide for $1 \mathrm{~h}$ at room temperature, then dehydrated in a growing gradient of ethanol and embedded in 812 epoxy resin (Polysciences, Inc., Warrington, PA, USA). Subsequently, $70 \mathrm{~nm}$ sections were cut on an ultra-microtome (SuperNova; LKB, Bromma, Sweden). The sections were post-stained with $2 \%$ uranyl acetate for 15 min and Reynald's lead citrate for $15 \mathrm{~min}$. The sections were imaged at $120 \mathrm{kV}$ on a Tecnai G20 TEM (FEI, Hillsboro, OR, USA).

Statistical analysis. The Student-Newman-Keuls multiple comparison test, following either one-way analysis or Student's $\mathrm{t}$-test, was performed to evaluate the statistical significance with Sigma Plot 10.0 software (Systat Software, Inc., Chicago, IL, USA). $\mathrm{P}<0.05$ was considered to indicate a statistically significant difference.

\section{Results}

MSCs at higher passage exhibit senescent characteristics. Cells with colony-forming growth and fibroblast-like morphology were derived from passage 2 (P2). Proliferation was gradually reduced over the course of long-term cultivation until the cells became enlarged and flat, and stopped proliferating at P6. The cells at this stage exhibited significantly increased SA- $\beta$-gal activity, a biomarker of senescent cells in culture, with a $>90 \%$ positive stain (Fig. 1A-C). Therefore, the cells were termed senescent MSCs. In addition, p16 and p21, senescence-related molecules, were markedly 
A

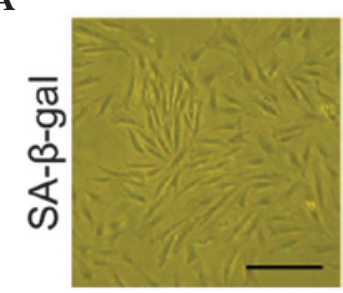

P2

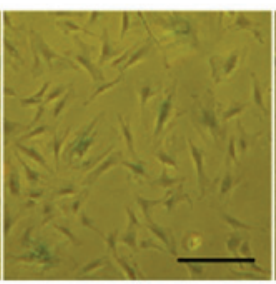

P4

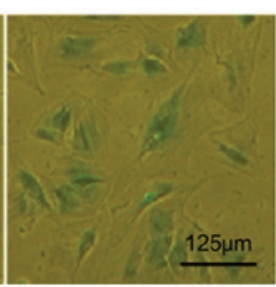

P6
B

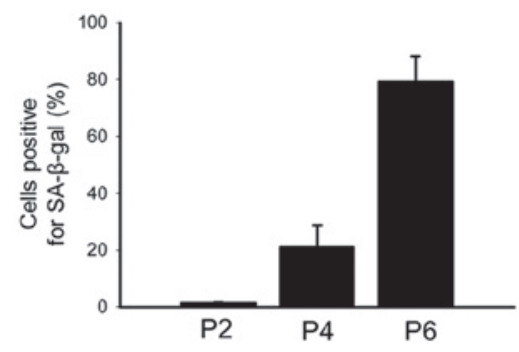

D

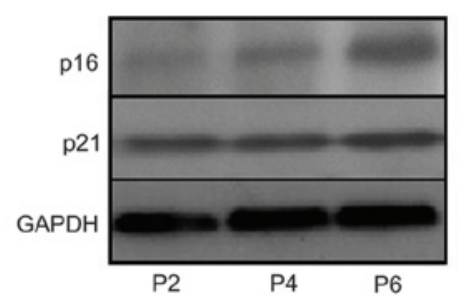

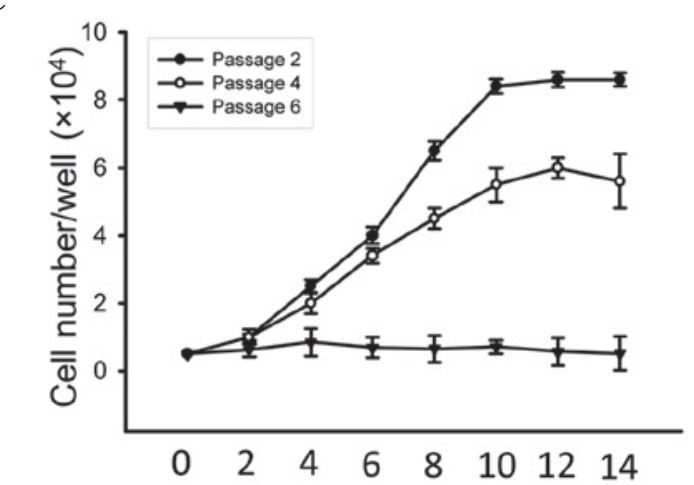

Figure 1. Senescence characteristics of rat mesenchymal stem cells (MSCs) were determined. (A) Senescence $\beta$-galactosidase (SA- $\beta$-gal) expression levels in MSCs were detected at different passages (P). (B) Histogram showing senescent cells as a percentage of the total population; $\mathrm{P}<0.05$, MSCs at P6 compared with MSCs at P2; n=4. (C) Growth profiles of MSCs at P2, P4 and P6 were determined by cell counting. (D) The expression levels of senescence-related proteins $\mathrm{p} 16$ and $\mathrm{p} 21$ were increased in $\mathrm{p} 4$ and $\mathrm{p} 6$, compared with those at $\mathrm{P} 2$.

A
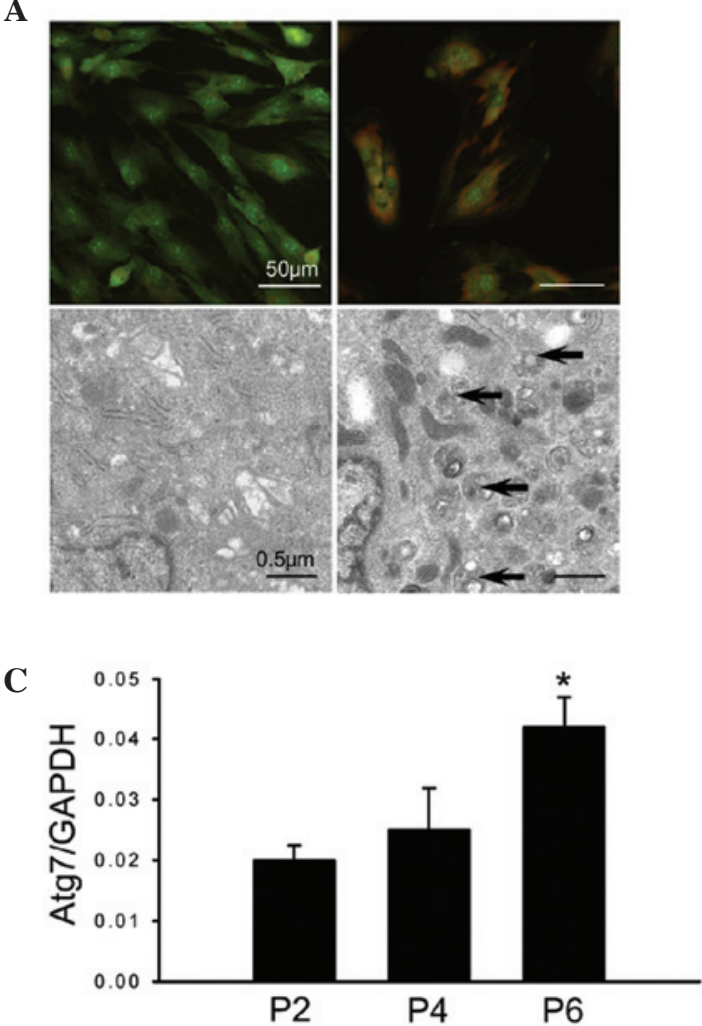

B
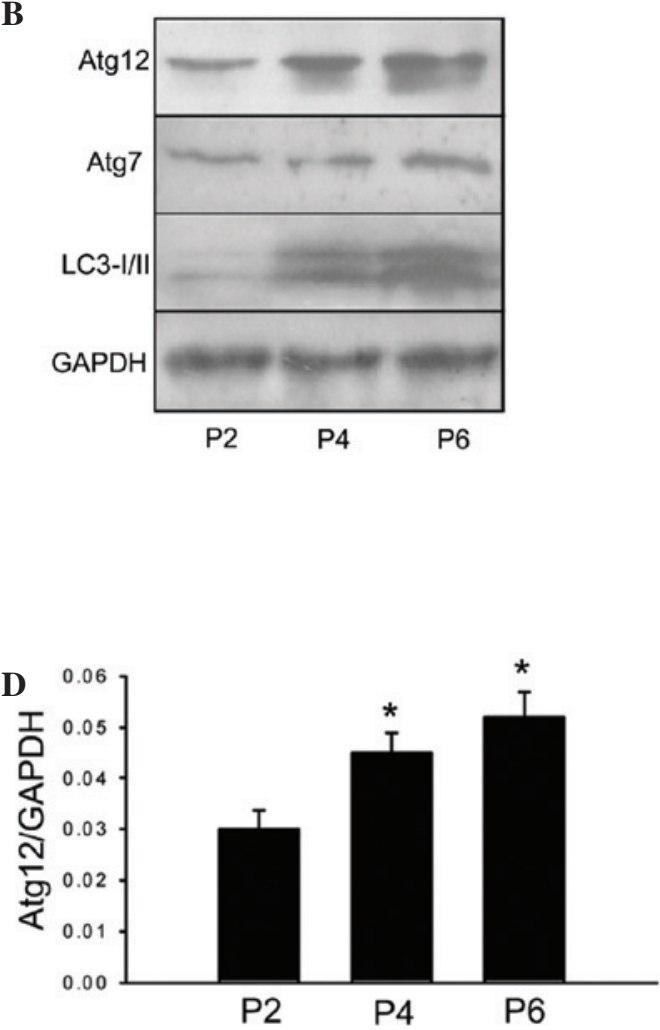

Figure 2. Autophagic activity in rat mesenchymal stem cells (MSCs) was determined in parallel with senescence detection. (A) Cells were stained with acridine orange and imaged by fluorescence microscopy (upper panel); red, acidic vesicular organelles; green, nuclei; bright green, cytoplasm. Autophagic vacuoles were observed by transmission electron microscopy at P2 (lower left panel) and P6 (lower right panel). Black arrows indicate autophagosome formation (magnification, x 5.000). (B) Immunoblot analysis of Atg12, Atg7 and microtubule-associated protein 1A/1B-light chain 3 (LC3-I/II) protein expression levels in MSCs at passages P2, P4 and P6. GAPDH served as an internal control. (C and D) Atg7 and Atg12 gene expression levels were assessed by reverse transcription polymerase chain reaction. ${ }^{*} \mathrm{P}<0.05$, compared with $\mathrm{MSC}$ at $\mathrm{P} 2 ; \mathrm{n}=6$. 

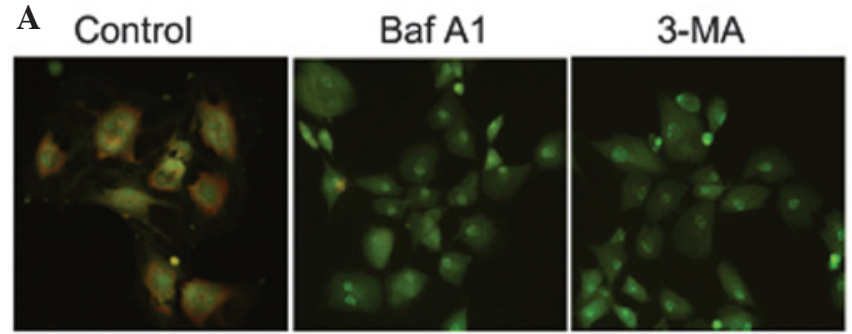

B

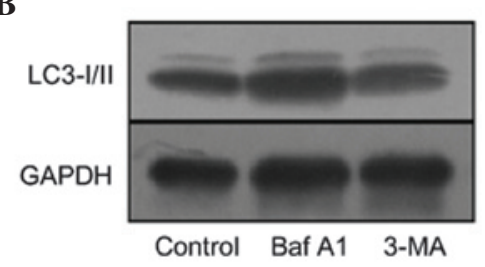

Figure 3. Effects of 3-methyladenine (3-MA) and Bafilomycin A1 (Baf A1) on the autophagic activity of rat mesenchymal stem cells (MSCs). (A) Detection of acidic vesicular organelles in treated MSCs using acridine orange. (B) Immunoblot analysis of microtubule-associated protein 1A/1B-light chain 3 (LC3-I/II). GAPDH served as an internal control.

upregulated in senescent MSCs compared with MSCs at P2 (Fig. 1D).

Autophagic activity is upregulated during MSC senescence. To evaluate autophagic activity in senescent MSCs, autophagy was investigated by TEM, acridine orange staining and analysis of the levels of autophagy-related gene expression. Autophagic vacuoles were directly visualized by TEM. Autophagic vacuoles are identified by a double-membrane structure containing undigested cytoplasmic material, including organelles, as described by Mizushima et al (13). In the present study, senescent MSCs exhibited a greater number of autophagic vacuoles, predominantly located on one side of the nucleus, while MSCs at P2 exhibited a normal organization with a typical nucleus and a dense cytoplasm (Fig. 2A). Autophagy may also be demonstrated through the detection of autolysosomes, which are AVOs. Acridine orange-stained AVOs exhibted red punctation, with the nucleus stained green and the cytoplasm bright green. AVOs were observed in the MSCs at P2 and the senescent MSCs (Fig. 2A). LC3-II is closely associated with the autophagosomal membrane; monitoring LC3-II is widely used to assay the autophagic activity in cells. Accumulation of LC3-II indicates an elevated autophagic activity in senescent MSCs (Fig. 2B). Two other autophagy-associated effectors, Atg7 and Atg12, were also investigated by western blotting and RT-PCR. Senescent MSCs exhibited significantly increased expression levels of Atg7 and Atg12 protein and mRNA, compared with MSCs at P2 (Fig. 2B-D). These results therefore indicate that senescent MSCs exhibit high autophagic activity.

Inhibition of autophagy alleviates the senescent state of MSCs. Following cellular adherence for three days at P6, the senescent MSCs were treated with autophagic inhibitors (10 mm 3-MA and $5 \mathrm{~nm}$ Baf A1) for three days. Both 3-MA and Baf A1 inhibited autophagy, as shown by acridine orange staining where the AVOs were significantly reduced by autophagy inhibitor treatment (Fig. 3A). In addition, the expression levels
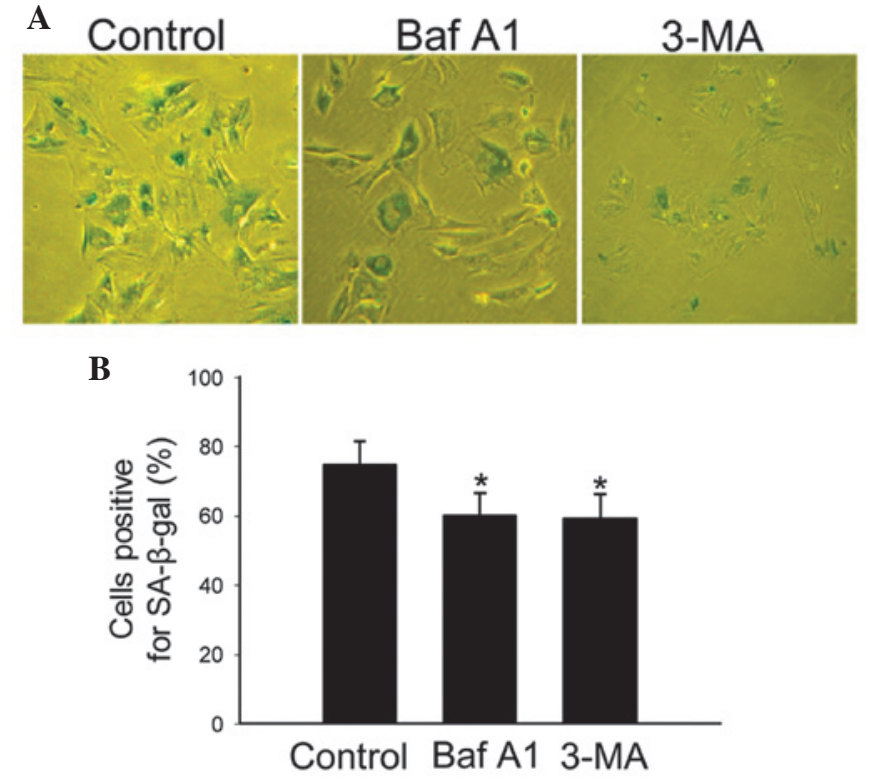

C

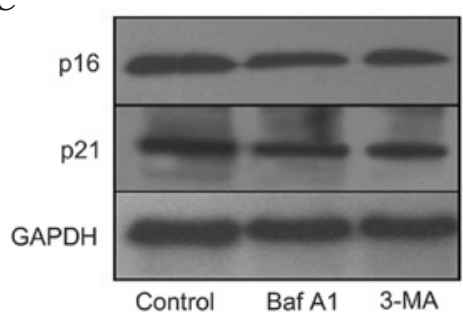

Figure 4. Effects of 3-methyladenine (3-MA) and Bafilomycin A1 (Baf A1) on mesenchymal stem cell senescence. (A) Cytochemical staining for senescence-associated $\beta$-galactosidase (SA- $\beta$-gal). (B) Histogram showing senescent cells as percentages of the total population. (C) Immunoblot analysis of p16 and p21 protein expression levels. GAPDH served as an internal control. ${ }^{*} \mathrm{P}<0.05$, compared with control group, $\mathrm{n}=6$.

of LC3 were reduced when senescent MSCs were treated with 3-MA (Fig. 3B). In Baf A1 treatment, autophagy was inhibited prior to autophagosome and lysosome fusion, thus LC3-II is not degraded through the fusion of autophagosomes and lysosomes (13). Therefore, in the MSCs, the LC3-II aggregation indicated that autophagy was suppressed.

Following confirmation that autophagy inhibitors efficiently suppress autophagy in senescent MSCs, the effect of these inhibitors on cell senescence was then analyzed. The rate of SA- $\beta$-gal-positive staining and the expression levels of senescence-related molecules were investigated to evaluate the senescent state of the cells treated with autophagy inhibitors. The rate of SA- $\beta$-gal-positive staining was significantly reduced following exposure to the autophagy inhibitors (Fig. 4A and B). Concomitant with the marked reduction in the activity of SA- $\beta$-gal, the expression levels of p16 and p21 proteins were also reduced in response to autophagy inhibitor treatment (Fig. 4C).

\section{Discussion}

MSCs have great potential in cell-based therapeutic applications and tissue engineering. However, due to the low cell frequencies in tissues and the restricted volume of obtainable tissues, MSCs 
are usually culture-expanded prior to applications (1,2). During long-term in vitro expansion, MSCs undergo a limited number of divisions and then cease proliferation. This is accompanied by a specific set of changes in cell physiology, morphology and gene expression, such as an enlarged and flattened morphology, increased SA- $\beta$-gal activity and the upregulation of senescence-related genes $(3,14)$. In accordance with these features, in the present study, rat MSCs exhibited senescent characteristics at P6. This is consistent with previous studies (10). Although the senescent cells enter a prolonged growth-arrested state, the cells remain metabolically active.

Autophagy, a predominant intracellular degradation catabolic process, is important for the maintenance of tissue homeostasis in stress conditions $(15,16)$. Although autophagy and senescence are generally considered to be two distinct cellular events, a number of studies have provided circumstantial evidence to indicate that the two are functionally intertwined. Sasaki et al (17) demonstrated that autophagy and senescence have been collaterally observed in the damaged bile ducts of patients with primary biliary cirrhosis. An increase in autophagic vacuoles was also evident in senescent endothelial cells (18), fibroblasts and human dental pulp cells $(19,20)$. Recently, Goehe et al (9) reported that autophagy and senescence commonly occur in parallel following the treatment of MCF-7 breast tumor cells with chemotherapeutic drugs. Furthermore, Young et al (21) suggested that autophagy mediates the acquisition of oncogene-induced senescence.

To examine the effects of autophagic activity on MSC senescence in the present study, autophagy was inhibited by pharmacological methods. The lack of highly specific autophagy inhibitors is recognized as a problem in autophagy research.3-MA, the most commonly used autophagy inhibitor, has been reported to inhibit the activity of phosphoinositide 3-kinase and block the formation of autophagosomes at the early stages of autophagy (22). Baf A1 alters lysosomal degradation by inhibiting acidification in later stages of autophagy (23). To avoid affecting other cell processes due to autophagic inhibition, 3-MA and Baf A1 were used as autophagy inhibitors in the present study. Attenuating autophagy activation contributed to reduced SA- $\beta$-gal activity and senescence-related molecule expression levels. However, a number of studies support an inverse association between autophagy and senescence $(24,25)$. This discrepancy may result from differences in experimental conditions; those studies analyzed basal autophagy, which was observed in the absence of internal or external stress.

The results from the present study have demonstrated that autophagy is involved in MSC senescence. This provides novel insights into understanding the underlying mechanism of MSC senescence. The functional senescence cells produce certain molecules that act in a positive feedback loop to reinforce the senescent phenotype and this constitutes a danger signal, which affects the microenvironment and sensitizes normal neighboring cells to senescence $(26,27)$. However, the efficient synthesis of these molecules requires autophagic protein degradation mechanisms (27). This indicates that autophagy modulates the functional activity of senescent MSCs. Results of the present study have shown that autophagy is activated during long-term MSC culture and a reduction in autophagic activity in senescent MSCs attenuates the senescent state. This result suggests that autophagic activity is a requirement for maintaining the senescent state of MSCs. The finding provides novel insights into the understanding of the senescent mechanism and a potential strategy for appropriate autophagy regulation that may counteract the senescence of MSCs. However, further studies are required in this field, particularly in the association between autophagy and senescence.

\section{Acknowledgements}

This study was supported by a grant from the National Natural Science Foundation of China (grant no. 81071269).

\section{References}

1. Wagner W and Ho AD: Mesenchymal stem cell preparations comparing apples and oranges. Stem Cell Rev 3: 239-248, 2007.

2. Nery AA, Nascimento IC, Glaser T, et al: Human mesenchymal stem cells: from immunophenotyping by flow cytometry to clinical applications. Cytometry A 83: 48-61, 2013.

3. Wagner W, Horn P, Castoldi M, et al: Replicative senescence of mesenchymal stem cells: a continuous and organized process. PLoS One 3: e2213, 2008.

4. Rodier F: Detection of the senescence-associated secretory phenotype (SASP). Methods Mol Biol 965: 165-173, 2013.

5. Narita M, Young AR and Narita M: Autophagy facilitates oncogene-induced senescence. Autophagy 5: 1046-1047, 2009.

6. Levine B and Klionsky DJ: Development by self-digestion: molecular mechanisms and biological functions of autophagy. Dev Cell 6: 463-477, 2004.

7. Levine B and Kroemer G: Autophagy in the pathogenesis of disease. Cell 132: 27-42, 2008

8. Gewirtz DA: Autophagy and senescence: a partnership in search of definition. Autophagy 9: 808-812, 2013.

9. Goehe RW, Di X, Sharma K, et al: The autophagy-senescence connection in chemotherapy: must tumor cells (self) eat before they sleep? J Pharmacol Exp Ther 343: 763-778, 2012.

10. $\mathrm{Hu} \mathrm{C}, \mathrm{Wu} \mathrm{Y}$, Wan Y, Wang Q and Song J: Introduction of hIGF-1 gene into bone marrow stromal cells and its effects on the cell's biological behaviors. Cell Transplant 17: 1067-1081, 2008.

11. Zheng Y, He L, Wan Y and Song J: H3K9me-enhanced DNA hypermethylation of the p16INK4a gene: an epigenetic signature for spontaneous transformation of rat mesenchymal stem cells. Stem Cells Dev 22: 256-267, 2013.

12. Chen Y, Azad MB and Gibson SB: Methods for detecting autophagy and determining autophagy-induced cell death. Can J Physiol Pharmacol 88: 285-295, 2010.

13. Mizushima N, Yoshimori $\mathrm{T}$ and Levine B: Methods in mammalian autophagy research. Cell 140: 313-326, 2010.

14. Bonab MM, Alimoghaddam K, Talebian F, et al: Aging of mesenchymal stem cell in vitro. BMC Cell Biol 7: 14, 2006.

15. Cuervo AM, Bergamini E, Brunk UT, et al: Autophagy and aging: the importance of maintaining 'clean' cells. Autophagy 1: 131-140, 2005.

16. Mizushima N: Autophagy: process and function. Genes Dev 21: 2861-2873, 2007.

17. Sasaki M, Miyakoshi M, Sato Y and Nakanuma Y: Autophagy mediates the process of cellular senescence characterizing bile duct damages in primary biliary cirrhosis. Lab Invest 90: 835-843, 2010.

18. Kurz DJ, Decary S, Hong Y and Erusalimsky JD: Senescence-associated (beta)-galactosidase reflects an increase in lysosomal mass during replicative ageing of human endothelial cells. J Cell Sci 113: 3613-3622, 2000.

19. Gerland LM, Peyrol S, Lallemand C, et al: Association of increased autophagic inclusions labeled for beta-galactosidase with fibroblastic aging. Exp Gerontol 38: 887-895, 2003.

20. Li L, Zhu YQ, Jiang L and Peng W: Increased autophagic activity in senescent human dental pulp cells. Int Endod J 45: 1074-1079, 2012.

21. Young AR, Narita M, Ferreira M, et al: Autophagy mediates the mitotic senescence transition. Genes Dev 23: 798-803, 2009. 
22. Fleming A, Noda T, Yoshimori T and Rubinsztein DC: Chemical modulators of autophagy as biological probes and potential therapeutics. Nat Chem Biol 7: 9-17, 2011.

23. Kaminskyy V and Zhivotovsky B: Proteases in autophagy. Biochim Biophys Acta 1824: 44-50, 2012.

24. Kang HT, Lee KB, Kim SY, Choi HR and Park SC: Autophagy impairment induces premature senescence in primary human fibroblasts. PLoS One 6: e23367, 2011.
25. Fujii S, Hara H, Araya J, et al: Insufficient autophagy promotes bronchial epithelial cell senescence in chronic obstructive pulmonary disease. Oncoimmunology 1: 630-641, 2012.

26. Rodier F and Campisi J: Four faces of cellular senescence. J Cell Biol 192: 547-556, 2011

27. Young AR and Narita M: Connecting autophagy to senescence in pathophysiology. Curr Opin Cell Biol 22: 234-240, 2010. 